\title{
A Note on Revenue Effects of Asymmetry in Private-Value Auctions
}

\author{
Todd R. Kaplan \\ Department of Economics \\ University of Exeter, \\ Streatham Court \\ Exeter, EX4-4PU UK \\ Dr@ToddKaplan.com
}

\author{
Shmuel Zamir \\ CNRS-EUREQua \\ Université Paris 1 Panthéon Sorbonne \\ Maison des Sciences Economiques \\ 106-112 Boulvard de l'Hopital \\ 75647 PARIS Cedex 13, France \\ LEI/CREST, France \\ and the Hebrew University, Israel \\ zamir@ensae.fr
}

February, 2002

\begin{abstract}
We formulate a way to study whether the asymmetry of buyers (in the sense of having different prior probability distributions of valuations) is helpful to the seller in private-value auctions (asked first by Cantillon [2001]). In our proposed formulation, this question corresponds to two important questions previously asked: Does a first-price auction have higher revenue than a second-price auction when buyers have asymmetric distributions (asked by Maskin and Riley [2000])? And does a seller enhance revenue by releasing information (asked by Milgrom and Weber[1982])? This is shown by constructing two Harsanyi games of incomplete information each having the same ex-ante distribution of valuations but in one beliefs are symmetric while in the other beliefs are sometimes asymmetric. Our main result is that answers to all three questions coincide when values are independent and are related when values are affiliated.
\end{abstract}

Keywords: asymmetric auctions, asymmetric beliefs, affiliation, linkage principle 
The purpose of this note is to suggest a way to formulate and study the question of whether asymmetry of buyers (in the sense of having different prior probability distributions of valuations) is helpful or harmful to the seller in private-value auctions. Do they generate higher or lower expected selling revenue than auctions in which buyers are symmetric?

The asymmetry in an auction (which treats the buyers equally such as the first or second price auction) can be in the distributions of the buyers' valuations or in the buyers' beliefs. The first point we make is that the only meaningful and interesting question about the effects on revenue is with respect to asymmetry of beliefs of the buyers. Distribution of valuations, whether symmetric or asymmetric, are part of the data of the situation. This probability distribution over the state of nature is not controlled by the seller or by us as analysts. Comparing an asymmetric auction to some benchmark symmetric auction (with a distribution based upon the asymmetric distributions), can easily lead to different results, depending on how this benchmark is chosen. Consider for example a two-buyer, first-price auction $(G, H)$ where the buyers' valuations $v_{1}$ and $v_{2}$ are independently drawn from the distributions $G$ and $H$, respectively. If $G$ and $H$ are the uniform distributions on $[0,1]$ and $[2,3]$, respectively, then clearly the asymmetric auction with $(G, H)$ generates higher revenue than the symmetric $(G, G)$ auction and lower than the symmetric $(H, H)$ auction. $^{1}$

The asymmetry of beliefs, on the other hand, are affected by the mechanism of the auction and may therefore in principle be manipulated by the seller via the informational structure of the auction. Therefore, our main methodological message in this note is:

\footnotetext{
1 The symmetric auctions $(G, G)$ and $(H, H)$ yield revenue of $1 / 3$ and $7 / 3$, respectively, while the asymmetric auction $(G, H)$ yields revenue of 1 (in equilibrium the second buyer bids 1 and wins).
} 
To study the effect of asymmetry in auctions, we have to single out the effect of asymmetry of beliefs by comparing two auctions using the same mechanism (first price, second price, etc.) and with the same joint distribution of valuations; they differ only in the mutual beliefs of the buyers about each other's valuations. These beliefs are symmetric in one auction and asymmetric in the other.

To illustrate this idea, consider an asymmetric auction $(G, H)$. We would like to study the effect of this asymmetry by considering the following two versions of ex-ante symmetric auctions.

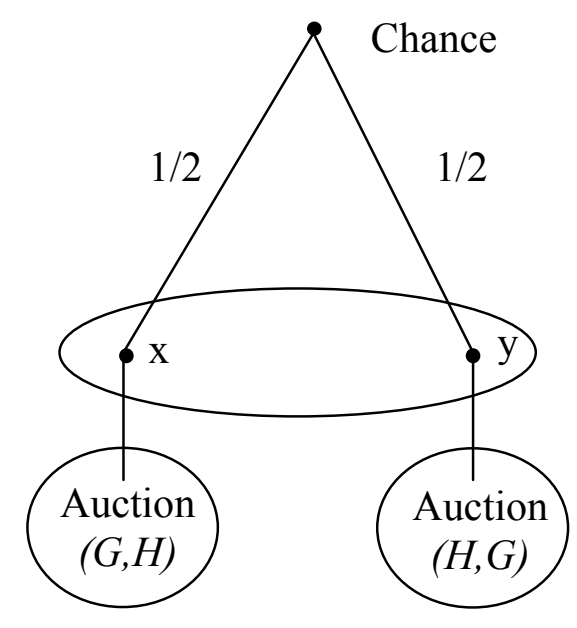

(s)

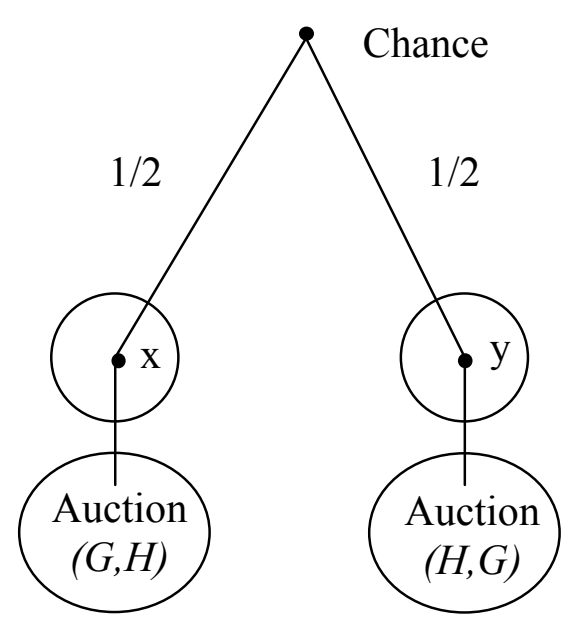

(a)

Figure 1: The symmetric game $(s)$ and the asymmetric game $(a)$.

In both versions, chance chooses with equal probability one of the pairs $(G, H)$ or $(H, G)$, then uses this pair to choose (independently) the valuations $\left(v_{1}, v_{2}\right)$ and inform each buyer of his value. However, while in $(s)$ no information is given to the buyers until they learn their values, in version $(a)$ both buyers are informed whether the first move by chance resulted in $x$ or in $y$. Clearly the $(a)$ version is equivalent to conducting 
one of the two asymmetric auctions $(G, H)$ or $(H, G)$ with equal probability. So exante the buyers are symmetric with distribution $F_{i}=\frac{1}{2} G+\frac{1}{2} H$. This is also the prior distribution of values for each of the two buyers in game $(s)$. Thus, any difference in revenue between $(s)$ and $(a)$ is due only to asymmetry in beliefs. However, such comparison is not straightforward since $(s)$ is not equivalent to a classic symmetric auction with distribution $\left(\frac{1}{2} G+\frac{1}{2} H, \frac{1}{2} G+\frac{1}{2} H\right)$. This is easily seen in that given his valuation $v_{i}$, buyer $i$ 's beliefs about the valuation of buyer $j$ is not $F_{j}$; it is another distribution resulting from Bayes' formula and depends on $v_{i}$. Thus, this is not the classic symmetric model of an auction with independent values in which beliefs of the buyers are common knowledge. ${ }^{2}$ So even though the two models differ only in the mutual beliefs of the buyers, this is not the comparison we would like to make, since we want to be able to compare standard symmetric and asymmetric auction games (as studied by Maskin and Riley [2000]) in which the beliefs of a buyer independent of his valuation.

To achieve this, we modify the two models in the following way: we consider a game in which a chance move first chooses a pair of distributions $\left(F_{1}, F_{2}\right)$ with the following probabilities.

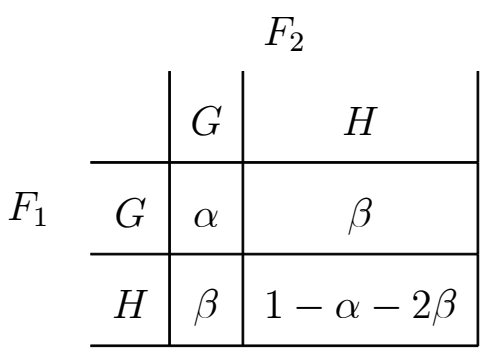

where $\alpha \geq 0, \beta \geq 0$ and $\alpha+2 \beta \leq 1$. Then the buyers' valuations $v_{1}$ and $v_{2}$ are 2 This is an asymmetric auction of the type studied by Landsberger et al. [2002], where the ranking of the valuations becomes common knowledge among buyers, the resulting situation is not the classical asymmetic auction model since the beliefs of the buyers are not common knowledge. 
drawn independently from the chosen $F_{1}$ and $F_{2}$, respectively.

This captures the previous games as a special case when $\alpha=0$ and $\beta=1 / 2$ and in addition allows for independent probability distributions when $\alpha=\mu^{2}$ and $\beta=\mu(1-\mu)$. This is the case where the buyers' values are drawn independently from $\left(F_{1}, F_{2}\right)$ which are chosen with probabilities: $P\left(F_{i}=G\right)=\mu ; \quad P\left(F_{i}=H\right)=1-\mu$, for $i=1,2$ and $0 \leq \mu \leq 1$. We henceforth call this the independent case.

We can now replace the previous games of $(s)$ and $(a)$ by $(\widehat{s})$ and $(\widehat{a})$ as shown in Figures 2 and 3. In these figures we make use of information set notation, that is, in $(\widehat{s})$ no buyer is informed of the outcome of the first chance move, while in $(\widehat{a})$ this chance move is revealed to both buyers.

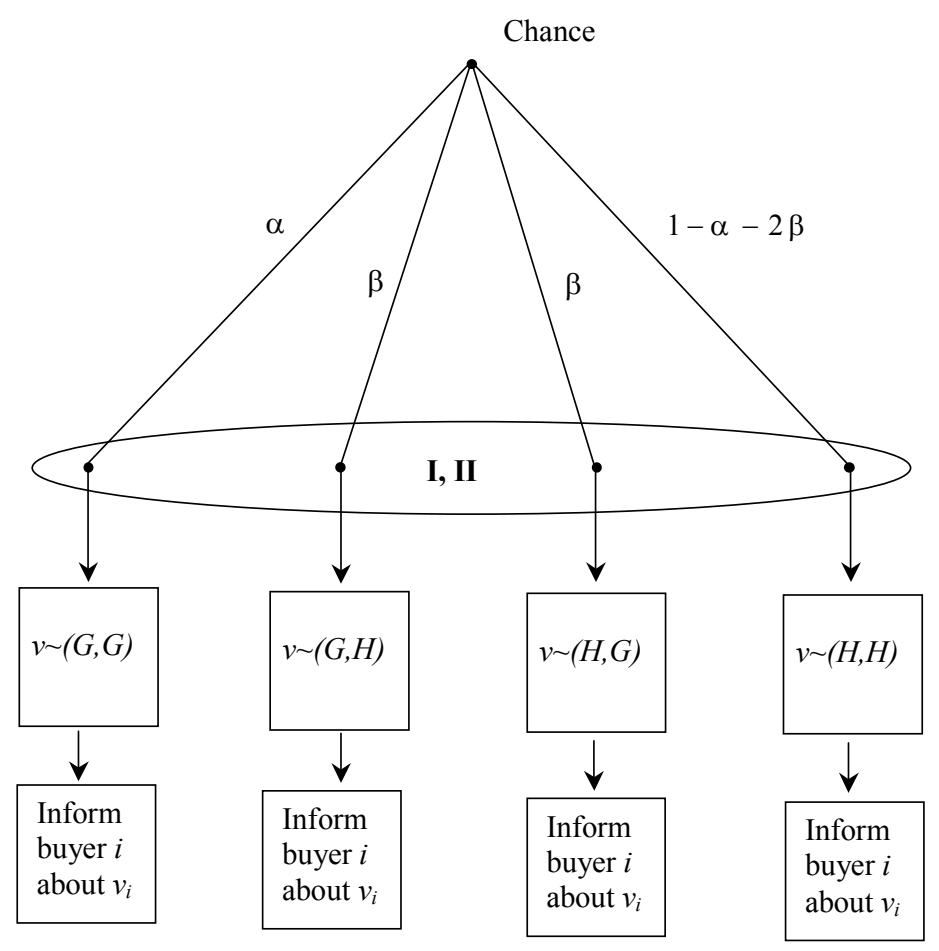

$(\hat{s})$

The symmetric game $(\widehat{s})$. 


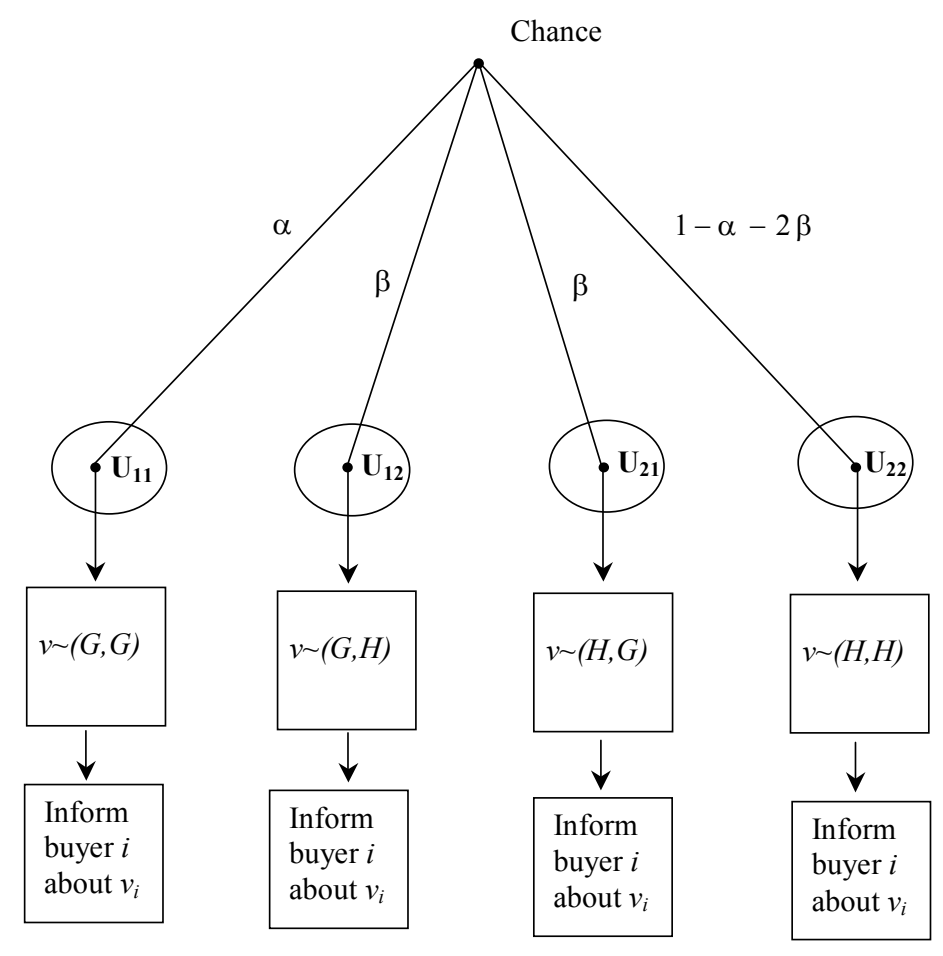

$(\hat{a})$

The asymmetric game $(\widehat{a})$.

We thus suggest to compare revenue in the independent case of the two games $(\widehat{s})$ and $(\widehat{a})$ : we will say that the asymmetry $(G, H)$ enhances (decreases) revenue if the revenue for game $(\widehat{a})$ is larger (smaller) than that of game $(\widehat{s})$.

- Note first that in the independent case, both games $(\widehat{s})$ and $(\widehat{a})$ have the same joint $i i d$ distribution of values (each $v_{i}$ is drawn from distribution $\mu G+(1-\mu) H$ ).

- Model $(\widehat{s})$ is a standard, symmetric, independent, private-value auction $(\mu G+$ $(1-\mu) H, \mu G+(1-\mu) H)$.

- Model ( $\widehat{a})$ leads (randomly) to one of the following standard independent value auctions: each is an independent, private-value auction with one of the following 
joint distributions: $(G, G),(H, H),(G, H)$ or $(H, G)$. The last two being standard, asymmetric, independent private-value auctions.

Using the aforementioned points, we can draw the following two observations about the two games.

Observation 1: In both games $(\widehat{s})$ and $(\widehat{a})$, for a second-price auction, it is a (weakly) dominant strategy to bid one's value. Thus, since the joint distribution of valuations is the same in $(\widehat{s})$ and $(\widehat{a})$, the distribution of the second highest valuation is the same and hence, the revenue of the seller is the same.

Observation 2: By revenue equivalence, in game $(\widehat{s})$ the revenue from the first-price and second-price auctions is the same.

Let us now use these observations to examine an asymmetric auction studied in the literature with an analytical solution.

Example 1. (Maskin \& Riley [2000]) Denote by $U[a, b]$ the uniform distribution on [a,b]. Let $G \sim U[0,1], H \sim U[2,3]$.

- Under the first-price auction in game $(\widehat{a})$, the seller's revenue is $(1 / 3) \mu^{2}+2 \mu(1-$ $\mu)+(7 / 3)(1-\mu)^{2}$.

- Under the second-price auction in game $(\widehat{a})$, the seller's revenue is equal to $(1 / 3) \mu^{2}$ $+\mu(1-\mu)+(7 / 3)(1-\mu)^{2}$ and is thus lower than revenue in the first-price 
auction.

- The seller's revenue in the second price auction is the expected second highest value. Hence, it is the same in games $(\widehat{a})$ and $(\widehat{s})$ since the distribution of valuations is the same (Observation 1).

- Since revenue is equivalent between first and second price auctions in game $(\widehat{s})$ (Observation 2), revenue in the first-price auction in game $(\widehat{s})$ is equal to revenue in the second-price auction of game $(\widehat{a})$ and thus lower than the revenue in a first-price auction in game $(\widehat{a})$.

- We conclude that revenue from a first-price auction is higher in game $(\widehat{a})$ than in game $(\widehat{s})$ : asymmetry increases revenue.

Note that the prior distribution in Harsanyi's model $(\mu, 1-\mu)$ does not affect the results as long as $0<\mu<1$. This is not a specificity of this example but rather a feature of the proposed approach. If the Harsanyi model is the right framework to make the comparison, then the result should not depend on the probability distribution of the information that renders the situation asymmetric. Whether the asymmetry of $(G, H)$ hurts or helps the seller should not depend upon its frequency of occurrence.

Example 2. Denote by $\delta(x)$ the degenerate distribution with mass one at $x$. Let $G$ $=\delta(3)$ and $H=\frac{1}{2} U[0,1]+\frac{1}{2} \delta(3) .{ }^{3}$

\footnotetext{
3 This example is inspired by an example in Maskin \& Riley [2000] where one buyer has a value of 2 and the other buyer half the time has a value of 0 and half the time has a value of 2 . We did not use their original example since if bids are restricted to be non-negative, that auction has no equilibrium.
} 
- In the equilibrium of the first-price auction in game $(\widehat{a})$, buyer 1 bids 1 with probability $1 / 2$ and with probability $1 / 2$ uses a mixed-strategy with cumulative distribution $W(b)=2 /(3-b)-1$ on $[1,2]$. Buyer 2 bids his value when it is less than or equal to 1 and when his value is 3 , he uses a mixed strategy with cumulative distribution $W(b)$.

- The expected revenue in the first-price auction of $(G, H)$ is $1.5 .^{4}$

- The expected revenue in the second-price auction of $(G, H)$ is 1.75 .

- Thus, the expected revenue in the first-price auction of game $(\widehat{a})$ is $3 \mu^{2}+1.5 \mu(1-$ $\mu)+\frac{13}{12}(1-\mu)^{2}$ and the expected revenue in the second-price auction of game $(\widehat{s})$ is $3 \mu^{2}+1.75 \mu(1-\mu)+\frac{13}{12}(1-\mu)^{2}$.

- Therefore, using similar logic as in example 1 , we conclude that revenue from a first-price auction is lower in game $(\widehat{a})$ than in game $(\widehat{s})$ : asymmetry reduces revenue..

The above two examples indicates an interesting relationship between two revenue comparisons. One (as done in Maskin \& Riley) is comparing the revenue between first and second price asymmetric auctions. The other (as addressed in Cantillon [2001]) is comparing the revenue in symmetric and asymmetric first-price auctions. By our approach, based on these two examples, we see that these two revenue comparisons are closely related: the asymmetry generates higher revenue in first-price auctions if and only if the asymmetric auction generates more revenue with a first-price auction than with a second-price auction. This is in fact a general result as established by the following proposition.

\footnotetext{
4 This is a derived by straightforward computation noticing that if $\mathbf{b}_{i} \sim W ; i=1,2$, and $\mathbf{b}_{i}$ are independent then $E\left[\mathbf{b}_{i}\right]=3-2 \operatorname{Ln}(2)$ and $E\left[\max \left\{\mathbf{b}_{1}, \mathbf{b}_{2}\right\}\right]=4 \operatorname{Ln}(2)-1$.
} 
Proposition 1: The asymmetry of $(G, H)$ enhances revenue of the seller (that is, revenue in $(\widehat{a})$ is higher than revenue in $(\widehat{s}))$ if and only if in the asymmetric auction $(G, H)$ the expected revenue from the first-price auction $R^{F P}(G, H)$ is higher than that of the second-price auction $R^{S P}(G, H)$.

Proof: Since in games $(\widehat{a})$ and $(\widehat{s})$ values are drawn from the same distribution, revenue from a second-price auction is the same in both games (Observation 1). In the game $(\widehat{s})$, the revenue equivalence holds due to independence. Hence, the revenue of a second-price auction in game $(\widehat{a})$ equals revenue of a first-price auction in $(\widehat{s})$ (Observation 2). Therefore, the ranking of revenue of the first price auction in $(\widehat{a})$ and $(\widehat{s})$ is the same as the ranking of revenue between first and second price auction in the game $(\widehat{a})$. To see this ranking observe that when beliefs are symmetric, the revenue is the same in first and second price auctions in the subgames of $(\widehat{a})$. Thus, any revenue difference between the first and second price auctions in game $(\widehat{a})$ comes only from the subgames when the beliefs are asymmetric, i.e. one buyer's value comes from $G$ and the other buyer's value comes from $H$ (and this is common knowledge). Therefore, if in this asymmetric case, the second-price auction has greater revenue than the first-price auction, the same revenue comparison is valid for the game $(\widehat{a})$, and vice versa. Therefore, the ranking of revenue between a first-price auction and a second-price auction in the asymmetric auction $(G, H)$ determines the ranking of revenue between a first-price auction in game $(\widehat{a})$ and a first-price auction in game $(\widehat{s})$.

Corollary: The revenue ranking of the symmetric auction $(\widehat{s})$ and the asymmetric 
auction $(\widehat{a})$ is the same for all $0 \leq \mu \leq 1$.

This result indicates that for an auction $(G, H)$ where the $R^{F P}(G, H)>R^{S P}(G, H)$, it is worthwhile for the seller to release information (about distributions of valuations) in the game $(\widehat{s})$ so as to generate an asymmetry of beliefs. Note by releasing or withholding information about the valuations, the seller does not affect the revenue in a second price auction, since this is determined only by the distribution of valuations which is not affected by these informational manipulations. Thus, in this case, the asymmetry created by this release of information is beneficial in a first-price auction and has no effect in a second-price auction. Likewise, when $R^{F P}(G, H)<R^{S P}(G, H)$, it is worthwhile for the seller to keep this information secret so as to maintain the symmetry of the buyers' beliefs. This is a result in the flavor of Milgrom \& Weber's [1982] linkage principle addressing the revenue effect of the seller releasing his private information.

This proposition, in conjunction with Maskin and Riley's results are in contrast with the conclusion of Cantillon [2001] that asymmetry is disadvantageous to the seller. As the above two examples show, the asymmetry $\left(F_{1}, F_{2}\right)$ may be advantageous or disadvantageous to the seller even if we restrict attention to CSD comparable distributions, i.e. $\left(F_{s}, F_{w}\right)$ such that $F_{s}$ conditionally stochastically dominates $F_{w} .{ }^{5}$

The difference in results follows clearly from the difference of the two approaches: Cantillon compares an asymmetric model $\left(F_{1}, F_{2}\right)$ with the benchmark symmetric model $\left(\sqrt{F_{1} F_{2}}, \sqrt{F_{1} F_{2}}\right)$. These two models do not correspond to the same joint distri-

\footnotetext{
5 Distribution $F_{s}$ conditionally stochastically dominates $F_{w}$ if for any $x<y$ (for which $F_{s}(x)$ and
} $\left.F_{s}(y) \in(0,1)\right)$, we have $\frac{F_{s}(x)}{F_{w}(x)}<\frac{F_{s}(y)}{F_{w}(y)}$ (see Maskin \& Riley 2000). 
bution of valuations. ${ }^{6}$ Hence, we argue, the difference between the two models is due to both the difference of value distributions and the difference in mutual beliefs.

To extend our results to the case where in the symmetric model $(\widehat{s}),\left(v_{1}, v_{2}\right)$ are not independent, we need to view $(\widehat{s})$ as a more general Milgrom \& Weber model, which involves the notion of affiliation:

The distribution of $\left(v_{1}, v_{2}\right)$ with positive density $f\left(v_{1}, v_{2}\right)$ is said to be (strictly) affiliated if for any $v=\left(v_{1}, v_{2}\right)$ and $\bar{v}=\left(\bar{v}_{1}, \bar{v}_{2}\right)$ the following inequality holds:

$$
f(v \vee \bar{v}) f(v \wedge \bar{v})>f(v) f(\bar{v})
$$

Where $\vee$ and $\wedge$ denote respectively the coordinate wise maximum and minimum of the two vectors. When the opposite inequality holds, we say that $\left(v_{1}, v_{2}\right)$ are (strictly) negatively affiliated. Although it was not mentioned explicitly in Milgrom \& Weber, it was noticed (see for example Mathews [1987]) that their results extend to the case of negative affiliation namely:

(Theorem 15 of Milgrom \& Weber) [With affiliation] the expected selling price in the second-price auction is at least as large as in the first-price auction.

(Extension of Theorem 15 of Milgrom \& Weber) With negative affiliation the expected selling price in the first-price auction is at least as large as in the second-price auction.

To address the issue of affiliation in our model $(\widehat{s})$, we make use of the notion of MLR (Monotone Likelihood Ratio). The distributions $(G, H)$ are related by MLR if $g(x) / h(x)$ is increasing or $h(x) / g(x)$ is increasing. We note that this relationship

\footnotetext{
6 The specific choice of the distribution $\sqrt{F_{1} F_{2}}$ was to guarantee the same distribution of the
} highest valuations and hence the same distribution of surplus in both models. However, for instance, the distribution of the second highest valuation is not the same. In fact as Cantillon shows, the expected revenue of the second-price auctions is not the same. 
implies CSD: if $g(x) / h(x)$ is increasing, then $G$ conditionally stochastically dominates $H$, which in turn implies $G$ stochastically dominates $H{ }^{7}$

Lemma If $(G, H)$ are related by $M L R$ then (in both models $(\widehat{a})$ and $(\widehat{s})$ )

(i) if $(\alpha+\beta)^{2}-\alpha<0$ then $\left(v_{1}, v_{2}\right)$ are affiliated.

(ii) if $(\alpha+\beta)^{2}-\alpha>0$ then $\left(v_{1}, v_{2}\right)$ are negatively affiliated.

(iii) $(\alpha+\beta)^{2}-\alpha=0$ if and only if $\left(v_{1}, v_{2}\right)$ are independent.

Proof: Let $\left(v_{1}, v_{2}\right)$ and $\left(\bar{v}_{1}, \bar{v}_{2}\right)$ s.t. $v_{1}>\bar{v}_{1}$ and $v_{2}<\bar{v}_{2}$. Assuming that $G$ and $H$ have positive densities $g$ and $h$ respectively, then the joint distribution of $\left(v_{1}, v_{2}\right)$ in game $(\widehat{s})$ (as well as in game $(\widehat{a}))$ is $f\left(v_{1}, v_{2}\right)$ given by

$$
f\left(v_{1}, v_{2}\right)=\alpha g\left(v_{1}\right) g\left(v_{2}\right)+\beta g\left(v_{1}\right) h\left(v_{2}\right)+\beta h\left(v_{1}\right) g\left(v_{2}\right)+(1-\alpha-2 \beta) h\left(v_{1}\right) h\left(v_{2}\right)
$$

A sufficient condition for strict affiliation is

$$
f\left(v_{1}, \bar{v}_{2}\right) f\left(\bar{v}_{1}, v_{2}\right)>f\left(v_{1}, v_{2}\right) f\left(\bar{v}_{1}, \bar{v}_{2}\right)
$$

which yields through straightforward manipulation

$$
\left((\alpha+\beta)^{2}-\alpha\right)\left[g\left(\bar{v}_{1}\right) h\left(v_{1}\right)-g\left(v_{1}\right) h\left(\bar{v}_{1}\right)\right] \cdot\left[g\left(\bar{v}_{2}\right) h\left(v_{2}\right)-g\left(v_{2}\right) h\left(\bar{v}_{2}\right)\right]>0
$$

When $(G, H)$ are related by MLR, in the above inequality the two expressions in $[\cdots][\cdots]$ have opposite signs, hence when $(\alpha+\beta)^{2}-\alpha<0$ the inequality holds.

7 To see that MLR is stronger than CSD consider the following example of distributions on $[0,1]$ : $h(x)=1$ and $g(x)=3 x$ for $x \leq 1 / 2$ and $g(x)=2-x$ for $x>1 / 2$. Note that $H(x) / G(x)$ is increasing, but $h(x) / g(x)$ is not increasing. 
Likewise, the condition for strict negative affiliation is just the reverse sign of affiliation

$$
f\left(v_{1}, \bar{v}_{2}\right) f\left(\bar{v}_{1}, v_{2}\right)<f\left(v_{1}, v_{2}\right) f\left(\bar{v}_{1}, \bar{v}_{2}\right)
$$

Which is true when $(G, H)$ are related by MLR and $(\alpha+\beta)^{2}-\alpha>0$. Part (iii) holds since $(\alpha+\beta)^{2}-\alpha=0$ can be rewritten as $\beta=\sqrt{\alpha}-\alpha$ and if we let $\alpha=\mu^{2}$, then $\beta=\mu(1-\mu)$.

The comparison of first-price auction in $(\widehat{s})$ and $(\widehat{a})$ is now given by the following:

Proposition 2: If in games $(\widehat{s})$ and $(\widehat{a}), G$ and $H$ are $M L R$ then:

(i) if $(\alpha+\beta)^{2}-\alpha>0$ and $R^{F P}(G, H)<R^{S P}(G, H)$ then the first price revenue in $(\widehat{a})$ is lower than the first-price revenue in $(\widehat{s})$, that is $R^{F P}(\widehat{a})<R^{F P}(\widehat{s})$ (and thus is to the advantage of the seller to reveal information in order to induce such asymmetric beliefs).

(ii) if $(\alpha+\beta)^{2}-\alpha<0$ and $R^{F P}(G, H)>R^{S P}(G, H)$ then the first price revenue in $(\widehat{a})$ is higher than the first-price revenue in $(\widehat{s})$, that is $R^{F P}(\widehat{a})<R^{F P}(\widehat{s})$ (and thus is to the advantage of the seller to keep such information secret).

(iii) if $(\alpha+\beta)^{2}-\alpha=0$ and $R^{F P}(G, H)(>,=,<) R^{S P}(G, H)$, then the first price revenue in $(\widehat{a})$ is respectively $(>,=,<)$ the first-price revenue in $(\widehat{s})$ that is $R^{F P}(\widehat{a})(>,=$ $,<) R^{F P}(\widehat{s})$ (this is case was covered before and does not depend upon the relationship of $G$ and $H)$.

\section{Proof:}

Part (iii) is a restatement of Proposition 1, while parts $(i)$ and $(i i)$ are proved in a similar manner and sketched as follows $(\stackrel{M W}{\Longrightarrow}$ stands for implied by Milgrom \& Weber'): 


$$
\underbrace{(\alpha+\beta)^{2}-\alpha>0} \underbrace{G \text { and } H \text { are related by MLR }} \underbrace{(\alpha+\beta)^{2}-\alpha<0}
$$

$\left.\begin{array}{l}(1) \\ (2)\end{array}\right\} \Longrightarrow\left(v_{1}, v_{2}\right)$ are negatively affiliated $\stackrel{M W}{\Longrightarrow} R^{F P}(\widehat{s})>R^{S P}(\widehat{s})=R^{S P}(\widehat{a})$. So if $R^{F P}(G, H)<R^{S P}(G, H)$ then $R^{F P}(\widehat{a})<R^{S P}(\widehat{a}) \Longrightarrow R^{F P}(\widehat{s})>R^{F P}(\widehat{a})$.

$\left.\begin{array}{l}\text { (2) } \\ \text { (3) }\end{array}\right\} \Longrightarrow\left(v_{1}, v_{2}\right)$ are affiliated $\stackrel{M W}{\Longrightarrow} R^{F P}(\widehat{s})<R^{S P}(\widehat{s})=R^{S P}(\widehat{a})$.

So if $R^{F P}(G, H)>R^{S P}(G, H)$ then $R^{F P}(\widehat{a})>R^{S P}(\widehat{a}) \Longrightarrow R^{F P}(\widehat{s})<R^{F P}(\widehat{a})$.

Note that the above proposition covers only part of the possible cases: no general conclusion can be made for the cases $\left(i^{\prime}\right)$ if $(\alpha+\beta)^{2}-\alpha>0$ and $R^{F P}(G, H)>$ $R^{S P}(G, H)$ and $\left(i i^{\prime}\right)$ if $(\alpha+\beta)^{2}-\alpha<0$ and $R^{F P}(G, H)<R^{S P}(G, H)$. It is plausible that in these cases, the ranking can be in either direction.

\section{Concluding Remarks}

Among the interesting questions asked regarding the seller revenue in auctions are the following:

1. Is the asymmetry of buyers distributions helpful or harmful to the seller (asked by Cantillon [2001])?

2. When the buyers have asymmetric distributions is the first-price auction better than the second price auction (asked by Maskin and Riley [2000])? 
3. Can a seller enhance his revenue by releasing information (Milgrom and Weber $[1982]) ?$

In this paper we addressed the first question and made the point that the appropriate comparison of revenue should be between models with the same distribution of values but one symmetric in beliefs while the other asymmetric in beliefs. An example of such an asymmetric model is provided in Landsberger et al. [2002] where the asymmetry of beliefs is generated by making common knowledge the ranking of the buyers' valuations. $^{8}$

To study the effect of the asymmetry of two distribution of valuations $(G, H)$, we propose two Harsanyi game models of incomplete information $(\widehat{a})$ and $(\widehat{s})$. Since by construction the two models have the same distributions of valuations, the second-price auction has the same revenue in both games, we thus use the first-price auction for the revenue comparison. In doing so, we find that the results hinge on the answer to the above second question. Furthermore, since the release of information to the buyers in the model $(\widehat{s})$ induces the model $(\widehat{a})$, the results are naturally related to the third question, namely, if this additional information is known by the seller. ${ }^{9}$

\footnotetext{
8 For a class of initial symmetric distributions (including the uniform), they found that in the first-price auction this asymmetry enhances revenue.

9 In Kaplan and Zamir [2000], we ask the additional related question as to whether an informed seller would use his information strategically; that is, if the seller were considered part of the game, then would he still release information in equilibrium.
} 


\section{References}

Cantillon, E. (2001): “The Effect of Bidders' Asymmetries on Expected Revenue in Auctions," CEPR Discussion Paper DP2675.

KAPlan, T., and S. ZAmiR (2000): "The Strategic Use of Seller Information in Private-Value Auctions," Hebrew University of Jerusalem Discussion Paper - 221.

Landsberger, M., J. Rubinstein, E. Wolfstetter, and S. Zamir (2002): "First-Price Auctions When the Ranking of Valuations is Common Knowledge," Forthcoming in Review of Economic Design, available as Hebrew University of Jerusalem Discussion Paper - 117, September 1996.

Maskin, E., and J. Riley (2000): "Asymmetric Auctions," Review of Economic Studies, 67(3), 413-438.

Matthews, S. (1987): “Comparing Auctions for Risk Averse Buyers: A Buyer's Point of View," Econometrica, 55(3), 633-646.

Milgrom, P. R., and R. J. Weber (1982): "A Theory of Auctions and Competitive Bidding," Econometrica, 50(5), 1089-1122. 